\title{
Communication-Neutron Radiography of the Water/Gas Distribution in the Porous Layers of an Operating Electrolyser
}

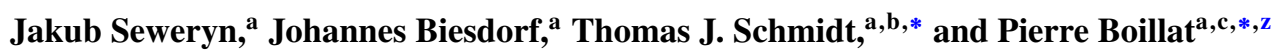 \\ ${ }^{a}$ Electrochemistry Laboratory, Paul Scherrer Institut, CH-5232 Villigen, Switzerland \\ ${ }^{b}$ Laboratory of Physical Chemistry, ETH Zürich, CH-8093 Zürich, Switzerland \\ ${ }^{c}$ Neutron Imaging and Activation Group, Paul Scherrer Institut, CH-5232 Villigen, Switzerland
}

\begin{abstract}
Neutron radiography was applied to image the water-gas distribution in an operating small scale PEM electrolyser, building up on the anisotropic resolution improvements previously developed for fuel cell imaging. The ability of neutrons to image this distribution across porous materials made of titanium was demonstrated for the first time. This paper presents the procedure's description and limitations, focusing on water content in the porous layers as a function of distance from electrode. A surprising pattern of water content in anodic porous material was observed, which sheds light on the topic of mass transport limitations in PEM electrolysers. (C) The Author(s) 2016. Published by ECS. This is an open access article distributed under the terms of the Creative Commons Attribution 4.0 License (CC BY, http://creativecommons.org/licenses/by/4.0/), which permits unrestricted reuse of the work in any medium, provided the original work is properly cited. [DOI: 10.1149/2.0641607jes] All rights reserved.
\end{abstract}

Manuscript submitted December 14, 2015; revised manuscript received April 4, 2016. Published April 15, 2016. This paper is part of the JES Focus Issue on Electrolysis for Increased Renewable Energy Penetration.

The topic of water - gas transport inside proton exchange membrane (PEM) water electrolysers has not been widely studied in the past. Most of the attention was paid to channel regions, as they are relatively easy to access with both numerical simulations and imaging methods. For instance, the influence of gas bubbles on the velocity field in the flow channels has been modelled, together with simple experimental validation of overall pressure drop. ${ }^{1,2}$ On the size level of flow channels, incorporating transparent windows into the cell can enable visualization of bubbles and temperature distribution measurement, as shown by Dedigama et al. ${ }^{3}$ However, since this kind of modification usually allows observing only the area under the nonconductive window, its representativeness is questionable. This can be overcome using X-ray radiography, as reported by Hoeh et al. ${ }^{4}$ Still, none of the work accesses the porous layer, and therefore water content at the electrode-to-porous layer interface is unknown.

To fully address the mass-transport limitations in PEM electrolysers, investigations should include the transport inside the porous layers. It has been shown, ${ }^{5}$ that the choice of porous material can influence the overall performance of an electrolysis cell. Still it is difficult to explain these results, relying solely on the measured electrical parameters of the cell.

Neutron radiography can be used to image and quantify the water/gas fraction inside the porous layers of an operating electrolyser cell. Although the spatial and temporal resolutions are lower than those of X-ray radiography, neutron radiography has the key advantage that several metals, including titanium which is commonly used for porous layers in electrolysis cells, exhibit a very good transparency. ${ }^{6}$ The scope of the work by Selamet et al. was however limited: not only a very specific design was used (no channels, Ti mesh for simultaneous optical and neutron imaging) but also the imaging orientation was limited to through plane imaging (neutron beam perpendicular to the membrane), which does not provide insight into the gas/water distribution across the different cell layers.

Here, we demonstrate the potential of neutron radiography to investigate water - gas transfer in porous layers by performing imaging of an operating electrolyser cell in in-plane (beam parallel to the membrane) configuration. For this purpose, we applied the anisotropic imaging technique which was established for fuel cells, ${ }^{7-13}$ including the tilted-detector setup, ${ }^{8}$ adapted for application to a small scale PEM electrolysis cell.

\section{Experimental}

Cell design.-The cell design is shown in Fig. 1. The active area $\left(1 \mathrm{~cm}^{2}\right)$ is in the center (Fig. 1a), elevated in order to remove remaining channels' length from the field of view. It has five flow channels, $10 \mathrm{~mm}$ long, $1 \mathrm{~mm}$ wide and $1 \mathrm{~mm}$ deep with a land width of $1 \mathrm{~mm}$. PTFE was used to fill the space around this elevation, as well as the gasket. The used porous media was sintered Ti powder (SIKA T5 from GKN) $10.0 \times 10.0 \times 1.2 \mathrm{~mm}$, porosity of approximately $30 \%$. A catalyst coated membrane (CCM) based on Nafion 117 membrane with $1 \mathrm{~cm}^{2}$ electrode was used (Greenerity E400 Gen. 1 from SolviCore).

Electrolysis operation.-Unless otherwise stated, the cell was operated at $50^{\circ} \mathrm{C}$ with a pressure of 2 bar $_{\text {abs }}$ on both sides. Water was fed to the anode side with a flow of $50 \mathrm{ml} \mathrm{min}^{-1}$ using a recirculation loop with gas removal. In order to prevent water from accumulating in the vicinity of the active area, the cathode site was purged with dry hydrogen at $800 \mathrm{Nml} \mathrm{min}{ }^{-1}$ in order to provide a defined boundary condition. The cell was operated in galvanostatic mode with simultaneous high-frequency-resistance (HFR) measurement at $5 \mathrm{kHz}$.

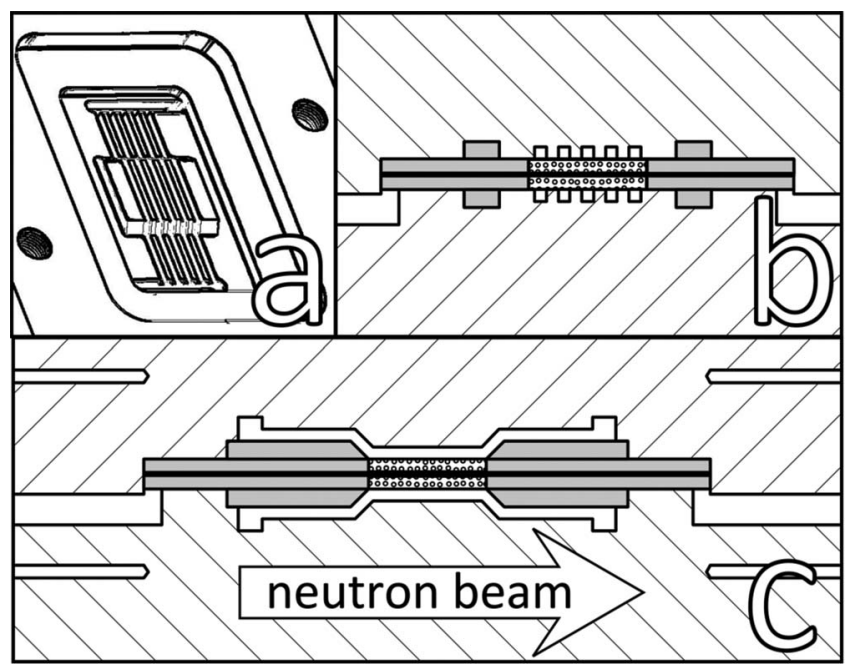

Figure 1. Cell design: Current collector with visible flow channels (a). Crosssection of assembled cell across (b) and along (c) the flow channels with marked neutron beam direction in in-plane mode. Dashed area represents $\mathrm{Al}$, gray PTFE, foam - like pattern Ti and black CCM. 


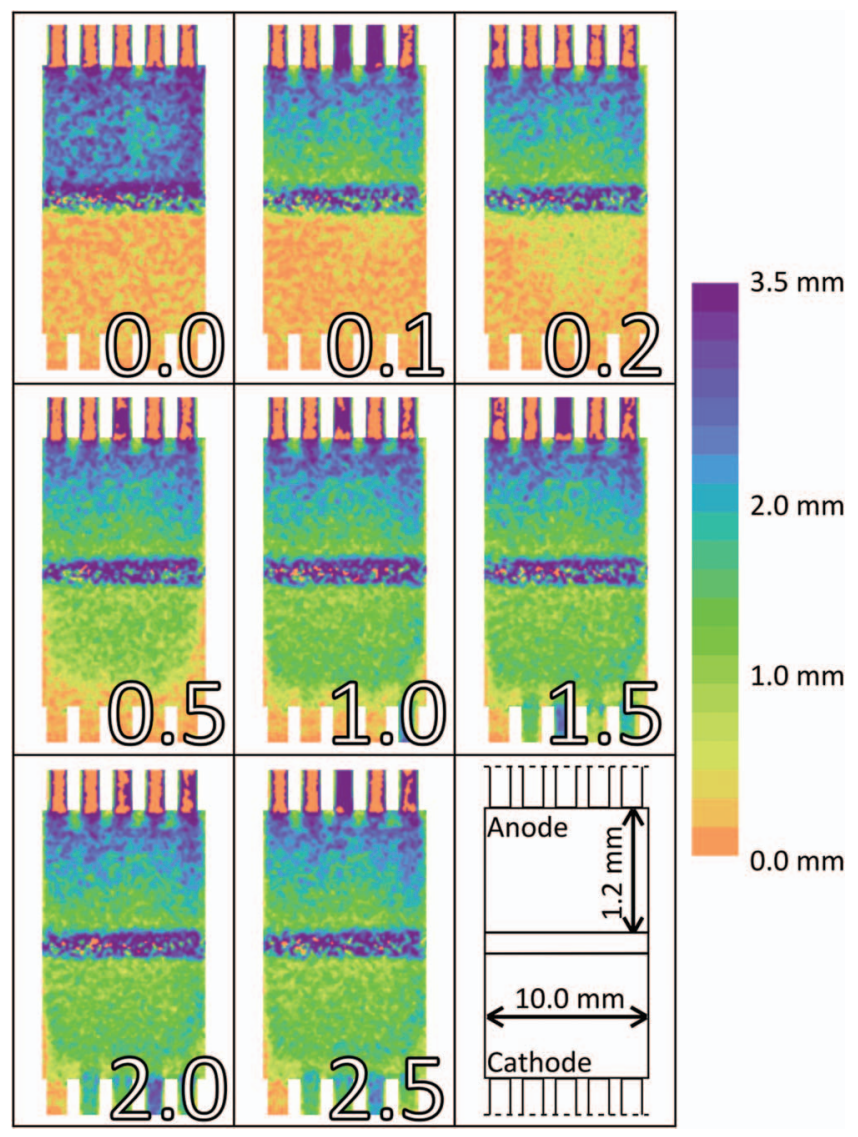

Figure 2. Neutron radiograms of PEM electrolyser obtained at varied current density (denoted in lower-left corner in $\mathrm{A} \mathrm{cm}^{-2}$ ). Color scale denotes water thickness. Images resulted from averaging over 5 minutes.

Imaging.-Imaging was performed at the Paul Scherrer Institute's SINQ neutron source at the NEUTRA thermal neutron radiography beamline. ${ }^{14}$ The investigated orientation was so-called in-plane setup (neutron beam parallel to membrane), with channels parallel to the beam, anode at the top. Images were taken with exposure time of 10 seconds and averaged over 5 minutes, in order to achieve sufficient signal-to-noise ratios. The cell operating parameters were kept constant for at least 5 minutes prior to imaging start. Images of the dry cell were taken as a reference for the image processing.

Calibration.-Calibration was performed according to the procedure described in Ref. 15 and the supplementary material; as a result the linear attenuation coefficient value was estimated to be $3.5 \mathrm{~cm}^{-1}$, which is in good agreement with the value previously stated for the NEUTRA beamline. ${ }^{9}$

\section{Results and Discussion}

Radiograms obtained in in-plane configuration (shown in Fig. 2) are the result of averaging over 5 minutes periods. The corresponding electrochemical data is shown in Fig. 3. Both anodic channels (upper borders) and the membrane (horizontal stripe in the middle) do not have sufficient transmittance for reliable analysis. The effective resolution in the vertical direction (across the membrane) was estimated to be approximately $75 \mu \mathrm{m}$.

Anodic porous layer.-When no current was applied to the cell, a uniform water distribution was quickly established in the anodic porous layer - this process was too fast to follow with the used exposure time.

After applying a current (the lowest examined current density was $0.1 \mathrm{~A} \mathrm{~cm}^{-2}$ ) a new pattern emerged. While in direction parallel-to-

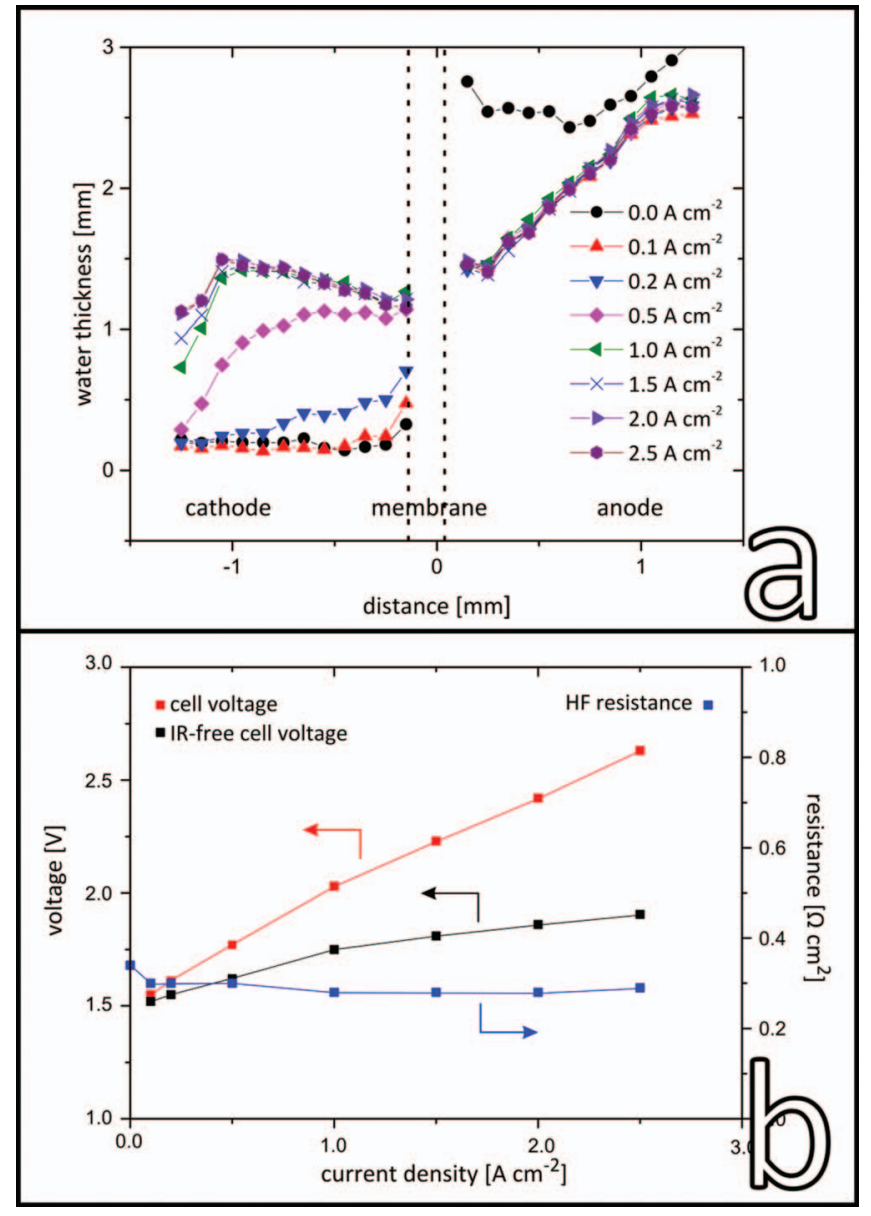

Figure 3. (a) Average water thickness across the cell. Area in the middle represents the membrane and due to the high noise to signal ratio its value has low practical meaning. Points on the left of it belong to cathodic porous layer while on the right to anodic. (b) Measured values of voltage and resistance, together with calculated IR-free resistance. The voltage at zero-current condition was unstable and therefore omitted.

membrane the water content was still fairly uniform (a weak ribchannel distribution can be observed at distances lower than $0.1 \mathrm{~mm}$ from the flow field), in orthogonal direction it is no longer valid. Instead, a linear gradient was established (Fig. 3), ranging from ca $85 \%$ (at the flow field) to $55 \%$ (at the membrane) of initial water thickness, independent on current density (the highest examined value was $2.5 \mathrm{~A} \mathrm{~cm}^{-2}$ ).

Cathodic porous layer.-In the lowest current cases, the measured water thickness is around $0.2 \mathrm{~mm}$, which is visible in Fig. 3. Attributing this to residual water presence is hardly possible, since the area of decreased transmission extends outside the porous layer. A plausible explanation is, that the reference images are biased by stronger background coming from scattering within the detector setup.

General.-We have found the observed patterns to be counterintuitive. The strong gradient on the anode side indicates a dynamic equilibrium between the transport of water to the electrode and the transport of gas away from the electrode. The fact that this equilibrium is unaffected by the magnitude of the flows over a wide range (from $0.1 \mathrm{~A} \mathrm{~cm}^{-2}$ to $2.5 \mathrm{~A} \mathrm{~cm}^{-2}$ ) is surprising. Because the heat production strongly varies with the current density, this also suggests that the thermal gradient does not play a major role in the water/gas distribution. The observed pattern clearly deserves attention as it can lead to the development of models for both transport in porous media and electro-osmotic drag. At all current densities, the water content in the vicinity of the electrode is approximately half of the initial value, 
which may suggest, that the water availability at the catalyst surface can be treated only on the microscale, with transport processes through the porous layer being neglected. Close to the channels, the attenuance was hardly distinguishable from the zero-current situation. This shows that water transport through this interface is fast. Contradicting conclusions were drawn in Ref. 3, where the dependence of the overpotential on the water flow rate was observed. These results were however obtained at different range of superficial water velocities, as well as different cell design, which may explain this discrepancy.

\section{Summary}

For the first time, neutron radiography was used to measure the water/gas distribution across the structure of an operating PEM electrolysis cell, with a focus of the water content within the porous media made of sintered titanium. The in-plane configuration was shown to give a detailed insight into the processes occurring in the porous media.

The observed pattern of water-gas distribution inside the porous media is surprising. The obtained gradient is unaffected by the local reactants' flow variation over a large range of current densities $(0.1 \mathrm{~A}$ $\mathrm{cm}^{-2}$ to $2.5 \mathrm{~A} \mathrm{~cm}^{-2}$ ), not showing any sign of water-starvation at high current; although not yet fully understood, these observation are an important starting point for the understanding and modelling of mass transport in PEM electrolysers.

\section{Acknowledgments}

We acknowledge the support from Swiss Federal Office of Energy through project 8100066 . TJS specifically thanks the Commission for
Technology and Innovation and the Swiss Competence Center for Energy Research (SCCER) Heat and Electricity Storage.

\section{References}

1. J. Nie, Y. Chen, S. Cohen, B. D. Carter, and R. F. Boehm, Int. J. Therm. Sci., 48, 1914 (2009)

2. J. Nie and Y. Chen, Int. J. Hydrogen Energy, 35, 3183 (2010).

3. I. Dedigama, P. Angeli, K. Ayers, J. B. Robinson, P. R. Shearing, D. Tsaoulidis, and D. J. L. Brett, Int. J. Hydrogen Energy, 39, 4468 (2014)

4. M. A. Hoeh, T. Arlt, I. Manke, J. Banhart, D. L. Fritz, W. Maier, and W. Lehnert, Electrochem. Commun., 55, 55 (2015).

5. S. A. Grigoriev, P. Millet, S. A. Volobuev, and V. N. Fateev, Int. J. Hydrogen Energy, 34, 4968 (2009).

6. O. F. Selamet, U. Pasaogullari, D. Spernjak, D. S. Hussey, D. L. Jacobson, and D. Mat, ECS Trans., 41, 349 (2011).

7. P. Oberholzer, P. Boillat, R. Siegrist, A. Kästner, E. H. Lehmann, G. G. Scherer, and A. Wokaun, Electrochem. Commun., 20, 67 (2012).

8. P. Boillat, G. Frei, E. H. Lehmann, G. G. Scherer, and A. Wokaun, Electrochem. Solid-State Lett., 13, B25 (2010).

9. A. Iranzo, P. Boillat, and F. Rosa, Int. J. Hydrogen Energy., 39, 7089 (2014).

10. J. D. Fairweather, D. Spernjak, J. Spendelow, R. Mukundan, D. S. Hussey, D. Jacobson, and R. L. Borup, ECS Trans., 58, 301 (2013).

11. N. Pekula, K. Heller, P. A. Chuang, A. Turhan, M. M. Mench, J. S. Brenizer, and K. Ünlü, Nucl. Instrum. Meth. A, 542, 134 (2005).

12. P. Boillat, P. Oberholzer, A. Kaestner, R. Siegrist, E. H. Lehmann, G. G. Scherer, and A. Wokaun, J. Electrochem. Soc., 159, F210 (2012).

13. I. Manke, Ch. Hartnig, M. Grünerbel, J. Kaczerowski, W. Lehnert, N. Kardjilov, A. Hilger, J. Banhart, W. Treimer, and M. Strobl, Appl. Phys. Lett., 90, 184101 (2007).

14. E. H. Lehmann, P. Vontobel, and L. Wiezel, Nondestruct. Test. Eval., 16, 191 (2001).

15. P. Boillat, J. Biesdorf, and P. Oberholzer, Electrochemistry Laboratory Annual Report 2013, p. 12 (2014). 